\title{
The Robust Ground Clutter Canceller Based on Inaccurate Prior Knowledge in Airborne Radar
}

\author{
Bo Dang $\mathbb{D}^{1}$ and Yan Zhou $\mathbb{D}^{2}$ \\ ${ }^{1}$ School of Electronic Engineering, Xi'an Shiyou University, Xi'an 710065, China \\ ${ }^{2}$ School of Information Science and Technology, Northwest University, Xi'an 710127, China \\ Correspondence should be addressed to Yan Zhou; spainraul123@126.com
}

Received 9 December 2020; Revised 20 June 2021; Accepted 22 August 2021; Published 7 September 2021

Academic Editor: Jean Jacques Loiseau

Copyright ( 2021 Bo Dang and Yan Zhou. This is an open access article distributed under the Creative Commons Attribution License, which permits unrestricted use, distribution, and reproduction in any medium, provided the original work is properly cited.

\begin{abstract}
Two-dimensional pulse-to-pulse canceller (TDPC) of ground clutter can effectively suppress the clutter along the clutter trace, and therefore the moving target detectability of the following space-time adaptive processing (STAP) algorithm can be improved after TDPC as the clutter prefilter. However, TDPC may greatly impair the energy of moving target when inaccurate knowledge is exploited, which is detrimental to target detection. Aiming at this problem, a robust two-dimensional pulse-to-pulse canceller (RTDPC) of ground clutter is proposed. In order to enhance the TDPC's robustness with inaccurate radar system parameters, which are mainly the platform velocity and crab angle, the errors of estimated platform velocity and crab angle are taken as the prior knowledge and added into the design of the clutter filter coefficient matrix. By exploiting RTDPC as the clutter prefilter, the moving target detectability of the following nonadaptive detection algorithm or STAP algorithm can also be enhanced. The simulated and MCARM data are utilized to verify the clutter suppression performance of RTDPC with inaccurate platform velocity and crab angle.
\end{abstract}

\section{Introduction}

For the fast moving platform, the clutter received by the airborne radar needs to be suppressed from the joint spatialtemporal domain, in which the clutter and moving target are separated. Space-time adaptive processing (STAP) has long been viewed as an effective tool for clutter suppression and target detection in airborne radar since it was first proposed by Brennan and Reed [1]. Two main limitations including computational cost and training sample demanding prevent it from being extensively applied and motivate the development of suboptimal dimension/rank-reduced STAP algorithms. By far, many literatures have studied various STAP methods to reduce the computational cost and training sample demanding in adaptive processing and enhance its practicability [2-4].

The dimension-reduced STAP methods [5-12], such as auxiliary channel processing (ACP) [5], joint domain localized (JDL) [6], and post-Doppler processing algorithms
$[7,8]$, can reduce the adaptive dimension and hence reduce the computational cost and the number of required training samples. On the other hand, the rank-reduced STAP methods [13-20], such as principle components (PC) [13], cross spectrum method (CSM) [16], and diagonal loading (DL) [17], make the eigendecomposition to the clutter plus noise covariance matrix (CPNCM) and try to estimate the clutter subspace. Although the rank-reduced STAP methods exploit the low rank property of clutter and reduce the number of training samples to twice of the clutter rank, they are computational intensive due to the CPNCM eigendecomposition. Besides, the eigenvectors of CPNCM cannot be accurately estimated when the number of training samples is small. Some target detection algorithms in airborne radar based on the reduced-rank STAP are soon proposed [21, 22]. Nowadays, the knowledge-aided (KA) radar, which can use the prior knowledge such as the digital elevation map (DEM) and geospatial databases to enhance STAP performance with small training sample support, has been attracting increasing 
attentions of researchers and practitioners [21-33]. In KA radar, various kinds of information are fused for predicting the clutter distribution. For example, the distribution of the ground clutter in the azimuth-Doppler domain can be determined in advance when the platform velocity and the crab angle, which can be obtained by the inertial navigation system (INS) and global positioning system (GPS), combined with radar operating parameters are known. References [34, 35] brought forward the method of twodimension pulse-pulse canceller (TDPC) of ground clutter based on this kind of prior knowledge, which is verified to be an effective clutter suppression method for airborne radar.

TDPC is in fact a nonadaptive clutter filter that takes the knowledge of radar operating parameters and platform velocity. However, due to the complex flight environment, the platform velocity and crab angle cannot be always correctly estimated. The inaccurate airborne platform velocity and crab angle used in the design of TDPC lead to the impairment to moving target and degradation of clutter suppression ability. Aiming at this problem, a robust twodimensional pulse-to-pulse canceller (RTDPC) of ground clutter is proposed. In order to enhance the TDPC's robustness with inaccurate prior knowledge that are mainly the platform velocity and crab angle, the errors of estimated airborne platform velocity and crab angle are added into the design of RTDPC. The moving target detectability of the following nonadaptive detection algorithm or STAP algorithm can also be enhanced. The simulated and MCARM data are utilized to verify the clutter suppression performance of RTDPC with inaccurate platform velocity and crab angle.

This paper is organized as follows. The ground clutter model and the original TDPC are reviewed in Section 2. Section 3 brings forward the principle of RTDPC. Section 4 illustrates the RTDPC filter cascaded with spatial-temporal matching (STM) or dimension-reduced STAP method. In Section 5, some experiments including simulated data and real data show the performance of RTDPC. Finally, we make a conclusion in Section 6.

\section{Ground Clutter Model and the Original TDPC}

The configuration of the airborne radar system to be studied is shown in Figure 1, which demonstrates the antenna array moving at a constant speed $v_{a}$ in the positive $x$-direction. The antenna array consists of $N$ array elements with interelement space $d$. The range cell under test (CUT) is uniformly divided into $N_{c}$ independent clutter patches without consideration of the earth curvature. The location of the $i$ th clutter patch is described by its azimuth angle $\theta_{i}$ and elevation angle $\varphi$. Assuming that the airborne radar system transmits $K$ pulses on the repetition of $T_{r}$ in one coherent processing interval $(\mathrm{CPI})$, the received clutter vector on the CUT at the $k$ th pulse is given by $[2,3]$ the following:

$$
\mathbf{x}(k)=\sum_{i=1}^{N_{c}} \alpha_{i} \exp \left(j 2 \pi(k-1) f_{d, i}\right) \mathbf{a}_{s}\left(\theta_{i}, \varphi\right), \quad k=1, \ldots, K .
$$

where $f_{d, i}=\left(2 T_{r} v_{a} \cos \left(\theta_{i}+\theta_{c}\right) \cos \varphi\right) / \lambda$ is the normalized Doppler frequency with $\theta_{c}$ being the crab angle, $\alpha_{i}$ is the echo amplitude, and $a_{s}\left(\theta_{i}, \varphi\right)$ is the spatial steering-vector and can be expressed as

$$
\mathbf{a}_{s}\left(\theta_{i}, \varphi\right)=\left[1, \exp \left(j 2 \pi f_{s, i}\right), \ldots, \exp \left(j 2 \pi(N-1) f_{s, i}\right)\right]^{T},
$$

in which $f_{s, i}=\left(d \cos \theta_{i} \cos \varphi\right) / \lambda$ is the normalized spatial frequency, $\lambda$ is the operating wavelength, and $(\cdot)^{T}$ is the transpose of a vector. The spatial-temporal snapshot of the clutter can be expressed as

$$
\mathbf{x}=[\mathbf{x}(1), \mathbf{x}(2), \ldots, \mathbf{x}(K)]+\mathbf{n}=\sum_{i=1}^{N_{c}} \alpha_{i} \mathbf{a}_{t}\left(f_{d, i}\right) \otimes \mathbf{a}_{s}\left(\theta_{i}, \varphi\right)+\mathbf{n},
$$

where $\quad \mathbf{a}_{t}\left(f_{d, i}\right)=\left[1, \exp \left(j 2 \pi f_{d, i}\right), \ldots, \exp (j 2 \pi(K-1)\right.$ $\left.\left.f_{d, i}\right)\right]^{T}$ is the temporal steering-vector and $\mathbf{n}$ is the white noise vector. One re-expresses (1) as the matrix-vector form as

$$
\mathbf{x}(k)=\mathbf{D F}(k) \boldsymbol{\alpha},
$$

where

$$
\begin{aligned}
\mathbf{D} & =\left[\mathbf{a}_{s}\left(\theta_{1}, \varphi\right), \mathbf{a}_{s}\left(\theta_{2}, \varphi\right), \ldots, \mathbf{a}_{s}\left(\theta_{N_{c}}, \varphi\right)\right], \\
\mathbf{F}(k) & =\operatorname{diag}\left(\left[\exp \left(j 2 \pi(k-1) f_{d, 1}, \ldots, \exp \left(j 2 \pi(k-1) f_{d, N_{c}}\right]\right),\right.\right. \\
\boldsymbol{\alpha} & =\left[\alpha_{1}, \ldots, \alpha_{N_{c}}\right]^{T} .
\end{aligned}
$$

In order to design TDPC, a coefficient matrix $\mathbf{A}_{\mathrm{TDPC}}$ is established to minimize the residual clutter energy between adjacent pulses, that is, as follows [35]:

$$
\min _{A}\|\boldsymbol{\varepsilon}(k)\|_{F}^{2}=\min _{A}\left\|\mathbf{A}_{\mathrm{TDPC}} \mathbf{x}(k)-\mathbf{x}(k+1)\right\|_{F}^{2}=\min _{A}\left\|\mathbf{A}_{\mathrm{TDPC}} \mathbf{D F}(k) \boldsymbol{\alpha}-\mathbf{D F}(k+1) \boldsymbol{\alpha}\right\|_{F}^{2},
$$

where $\|\cdot\|_{F}$ is the F-norm. Since the most of the energy contained in each $\mathbf{x}(k)$ comes from the clutter echo, the clutter can hence be suppressed by minimizing the difference in (6). Let $\bar{F}=\operatorname{diag}\left(\left[\exp \left(j 2 \pi f_{d, 1}, \ldots, \exp \left(j 2 \pi f_{d, N_{c}}\right]\right)\right.\right.$, we have that 


$$
\min _{A}\|\boldsymbol{\varepsilon}(k)\|_{F}^{2}=\min _{A}\left\|\mathbf{A}_{\mathrm{TDPC}} \mathbf{D} \bar{F}^{k-1} \boldsymbol{\alpha}-\mathbf{D} \overline{F F}^{k-1} \boldsymbol{\alpha}\right\|_{F}^{2}=\min _{A}\left\|\left(\mathbf{A}_{\mathrm{TDPC}} \mathbf{D}-\mathbf{D} \bar{F}\right) \bar{F}^{k-1} \boldsymbol{\alpha}\right\|_{F}^{2},
$$

which can be further described by the Cauthy-Shwarz in equation as

$$
\|\boldsymbol{\varepsilon}(k)\|_{F}^{2} \leq c\left\|\left(\mathbf{A}_{\mathrm{TDPC}} \mathbf{D}-\mathbf{D} \bar{F}\right)\right\|_{F}^{2},
$$

where $c=\left\|\bar{F}^{k-1} \alpha\right\|_{F}^{2}$ is a constant. Now, our goal is to minimize $\left\|\left(\mathbf{A}_{\mathrm{TDPC}} \mathbf{D}-\mathbf{D} \bar{F}\right)\right\|_{F}^{2}$. The following relation can be obtained after expanding $\left\|\left(\mathbf{A}_{\mathrm{TDPC}} \mathbf{D}-\mathbf{D} \bar{F}\right)\right\|_{F}^{2}$ :

$$
\begin{aligned}
& \left.\min _{A} \| \mathbf{A}_{\mathrm{TDPC}} \mathbf{D}-\mathbf{D} \bar{F}\right) \|_{F}^{2}=\min _{A} \operatorname{tr}\left[\left(\mathbf{A}_{\mathrm{TDPC}} \mathbf{D}-\mathbf{D} \bar{F}\right)^{H}\left(\mathbf{A}_{\mathrm{TDPC}} \mathbf{D}-\mathbf{D} \bar{F}\right)\right] \\
& =\min _{A} \operatorname{tr}\left(\mathbf{D}^{H} \mathbf{A}_{\mathrm{TDPC}}^{H} \mathbf{A}_{\mathrm{TDPC}} \mathbf{D}-\mathbf{D}^{H} \mathbf{A}_{\mathrm{TDPC}}^{H} \mathbf{D} \bar{F}-\bar{F}^{H} \mathbf{D}^{H} \mathbf{A}_{\mathrm{TDPC}} \mathbf{D}+\bar{F}^{H} \mathbf{D}^{H} \mathbf{D} \bar{F}\right) \\
& =\min _{A} f\left(\mathbf{A}_{\mathrm{TDPC}}\right),
\end{aligned}
$$

where $\operatorname{tr}(\cdot)$ is the trace of a matrix and $[\cdot]^{H}$ is the conjugate transpose of a matrix or a vector. Let the gradient of $f\left(\mathbf{A}_{\mathrm{TDPC}}\right)$ with respect to $\mathbf{A}_{\mathrm{TDPC}}^{H}$ be zero. Then, the solution to $(9)$ is

$$
\mathbf{A}_{\mathrm{TDPC}}=\mathbf{D} \bar{F} \mathbf{D}\left(\mathbf{D D}^{H}\right)^{-1} \cdot .
$$

The TDPC coefficient matrix $\mathbf{A}_{\mathrm{TDPC}}$ can be obtained if the matrices $\mathbf{D}$ and $\bar{F}$ are known, where $\mathbf{D}$ and $\bar{F}$ are only determined by the parameters defined in (5). Thus, if the parameters, such as platform velocity, crab angle, operating wavelength, and so on, are accurately known in advance, the matrices $\mathbf{D}$ and $\bar{F}$ can be utilized as the prior information for computing $\mathbf{A}_{\text {TDPC }}$. In other words, the TDPC coefficient matrix $\mathbf{A}_{\text {TDPC }}$ can be precalculated offline.

A prefiltering matrix of TDPC is devised as

$$
\mathbf{H}_{\mathrm{TDPC}}=\left[\begin{array}{cccccc}
\mathbf{A}_{\mathrm{TDPC}} & -\mathbf{I}_{N} & & & & \\
& \mathbf{A}_{\mathrm{TDPC}} & -\mathbf{I}_{N} & & \mathbf{0} & \\
& & \ddots & \ddots & & \\
& \mathbf{0} & & \mathbf{A}_{\mathrm{TDPC}} & -\mathbf{I}_{N} & \\
& & & & \mathbf{A}_{\mathrm{TDPC}} & -\mathbf{I}_{N}
\end{array}\right] \in \mathbf{C}^{(K-1) N \times K N},
$$

$$
\begin{aligned}
f_{d_{-} \text {est }} & =\frac{2\left(v_{a}+\Delta v_{a}\right) \cos \left(\theta+\theta_{c}+\Delta \theta_{c}\right) \cos \varphi}{\lambda f_{r}} \\
& =\frac{2 v_{a} \cos \theta_{r} \cos \Delta \theta_{c} \cos \varphi}{\lambda f_{r}}+\frac{2 \Delta v_{a} \cos \theta_{r} \cos \Delta \theta_{c} \cos \varphi}{\lambda f_{r}}-\frac{2 v_{a} \sin \theta_{r} \sin \Delta \theta_{c} \cos \varphi}{\lambda f_{r}}-\frac{2 \Delta v_{a} \sin \theta_{r} \sin \Delta \theta_{c} \cos \varphi}{\lambda f_{r}},
\end{aligned}
$$

where $\theta_{r}=\theta+\theta_{c}$ represents the real angle of a clutter patch with $\theta$ being the azimuth angle of a clutter patch and $\theta_{c}$ representing the crab angle. $\Delta \theta_{c}$ represents the estimated where $\mathbf{I}_{N}$ is a $N \times N$ identity matrix. Then, TDPC can suppress the clutter by the following equation:

$$
\bar{x}=\mathbf{H}_{\mathrm{TDPC}} \mathbf{x} .
$$

\section{Design of RTDPC}

From the aforementioned designing procedure, the effectiveness of TDPC depends heavily on the accuracy of prior knowledge. In fact, though some parameters, such as operating wavelength and elevation angle of CUT, can be accurately known, some other parameters, such as platform velocity and crab angle, can only be obtained with errors due to the inconstant aircraft control. As a consequence, inaccurate parameters exploited in the design will certainly influence the clutter filtering result of TDPC, and hence in this section, a robust TDPC based on the steering-vector selection will be designed to improve the effectiveness of the original TDPC with inaccurate parameters (mainly platform velocity and crab angle). To start with, the estimated platform velocity error and estimated crab angle error are assumed to be $\Delta v_{a}$ and $\Delta \theta_{c}$. Accordingly, the estimated normalized Doppler frequency can be expressed as crab angle error, namely, the error generated the estimation. For the first two items and last two items in (13), they can be further simplified as 


$$
\begin{aligned}
& \frac{2 v_{a} \cos \theta_{r} \cos \Delta \theta_{c} \cos \varphi}{\lambda f_{r}}+\frac{2 \Delta v_{a} \cos \theta_{r} \cos \Delta \theta_{c} \cos \varphi}{\lambda f_{r}}=f_{d} \cos \Delta \theta_{c}\left(1+\frac{\Delta v_{a}}{v_{a}}\right) \\
& -\frac{2 v_{a} \sin \theta_{r} \sin \Delta \theta_{c} \cos \varphi}{\lambda f_{r}}-\frac{2 \Delta v_{a} \sin \theta_{r} \sin \Delta \theta_{c} \cos \varphi}{\lambda f_{r}}=-\frac{2 v_{a} \sin \theta_{r} \sin \Delta \theta_{c} \cos \varphi}{\lambda f_{r}}\left(1+\frac{\Delta v_{a}}{v_{a}}\right) .
\end{aligned}
$$

For a typical airborne radar system, the accuracies for INU and GPS measuring velocity and crab angle are about $0.1 \mathrm{~m} / \mathrm{s}-0.2 \mathrm{~m} / \mathrm{s}$ and $1^{\circ}-1.5^{\circ}$, respectively. Generally, the velocity of airborne radar is over hundreds of meters per second. Therefore, reasonably speaking, $\left(\Delta v_{a} / v_{a}\right)$ should be a very small number and can be neglected in the Doppler calculation equation. Meanwhile, by using the facts that $\cos \Delta \theta_{c} \approx 1-\left(\Delta \theta_{c}^{2} / 2\right)$ and $\sin \Delta \theta_{c} \approx \Delta \theta_{c}$ when $\Delta \theta_{c}$ is very small, equation (14) can be further simplified as

$$
\begin{aligned}
f_{d_{-} \text {est }} & =\frac{2\left(v_{a}+\Delta v_{a}\right) \cos \left(\theta+\theta_{c}+\Delta \theta_{c}\right) \cos \varphi}{\lambda f_{r}} \approx f_{d} \cos \Delta \theta_{c}-\frac{2 v_{a} \sin \theta_{r} \cos \varphi}{\lambda f_{r}} \sin \Delta \theta_{c} \\
& \approx f_{d}\left(1-\frac{\Delta \theta_{c}^{2}}{2}\right)-\frac{2 v_{a} \sin \theta_{r} \cos \varphi}{\lambda f_{r}} \Delta \theta_{c},
\end{aligned}
$$

which becomes a equation related only with estimated crab angle, and the error between the true Doppler frequency $f_{d}$ and the estimated normalized Doppler frequency is $\Delta f_{d}=$ $f_{d}-f_{d_{\text {_ves }}}=f_{d}\left(\Delta \theta_{c}^{2} / 2\right)+\left(\left(2 v_{a} \sin \theta_{r} \cos \varphi_{l}\right) / \lambda f_{r}\right) \Delta \theta_{c}$. For verifying the feasibility of equation (15), the following results are demonstrated in Figure 2, where the average estimated Doppler frequency error (defined as $\sum_{i=1}^{N_{c}}\left|f_{d, i}-f_{d_{-} \text {est }, i}\right| / N_{c}$ ) curves in a range cell for $\Delta \theta_{c}=0.5^{\circ}, 1^{\circ}$, and $1.5^{\circ}$ by using equations (13) and (15) are separately plotted. Evidently, as the aforementioned analysis, the variation of $\Delta f_{d}$ with $\Delta v_{a}$ is negligible and the crab angle plays the dominated role in $\Delta f_{d}$. Consequently, when $\Delta \theta_{c}$ is determined, the upper bound of $\Delta f_{d}$ can be determined as $f_{d}\left(\Delta \theta_{c}^{2} / 2\right)+f_{d, \max } \Delta \theta_{c}$ for $\quad \Delta f_{d} \leq f_{d}\left(\Delta \theta_{c}^{2} / 2\right)+\left(2 v_{a} / \lambda f_{r}\right) \Delta \theta_{c}=f_{d}\left(\Delta \theta_{c}^{2} / 2\right)+$ $f_{d, \max } \Delta \theta_{c}$, where $f_{d, \text { max }}=\left(2 v_{a} / \lambda f_{r}\right)$ represents the maximum normalized Doppler frequency.

Let $\Delta f_{d, \max }=f_{d}\left(\Delta \theta_{c}^{2} / 2\right)+f_{d, \max } \Delta \theta_{c}$, then the estimated normalized Doppler frequency is located in the range $\left[f_{d}-\Delta f_{d, \max }, f_{d}+\Delta f_{d, \max }\right]$. Then, the normalized Doppler frequency span $\left[f_{d}-\Delta f_{d, \max }, f_{d}+\Delta f_{d, \max }\right]$ can be uniformly divided into $N_{d}$ samples, which are denoted as $f_{d, 1}^{\prime}, \ldots, f_{d, N_{d}}^{\prime}$. Accordingly, a spatial-temporal steeringvector dictionary is defined as

$$
\begin{aligned}
\mathbf{B}= & {\left[\mathbf{v}_{1}\left(\theta_{1}, \varphi, f_{d, 1}^{\prime}\right), \ldots, \mathbf{v}_{i}\left(\theta_{i}, \varphi, f_{d, j}^{\prime}\right), \ldots, \mathbf{v}_{N_{c}^{\prime}}\left(\theta_{N_{c}^{\prime}}, \varphi, f_{d, N_{d}}^{\prime}\right)\right], } \\
& i=1, \ldots, N_{c}^{\prime}, j=1, \ldots, N_{d}, \mathbf{B}=\left[\mathbf{v}_{1}\left(\theta_{1}, \varphi, f_{d, 1}^{\prime}\right), \ldots, \mathbf{v}_{i}\left(\theta_{i}, \varphi, f_{d, j}^{\prime}\right), \ldots, \mathbf{v}_{N_{c}^{\prime}}\left(\theta_{N_{c}^{\prime}}, \varphi, f_{d, N_{d}}^{\prime}\right)\right], \quad i=1, \ldots, N_{c}^{\prime}, j=1, \ldots, N_{d},
\end{aligned}
$$

where $\mathbf{B}$ is a full-column rank matrix, $N_{c}^{\prime}$ is the assumed number of clutter patches evenly distributed in the whole azimuth angle, and $\mathbf{v}_{i}\left(\theta_{i}, \varphi, f_{d, j}^{\prime}\right)=\mathbf{a}_{t}\left(f_{d, j}^{\prime}\right) \otimes \mathbf{a}_{s}\left(\theta_{i}, \varphi\right)$. After obtaining $\mathbf{B}$ and the snapshot in the CUT $\mathbf{x}$, a search method, which is similar to OMP [36], will be exploited to iteratively find the most relevant steering-vector to $\mathbf{x}$ from $\mathbf{B}$, and this subsection outlines the main steps.

Let $\mathbf{e}_{0}=\mathbf{x}$, in the first iteration, the most relevant steering-vector is selected as $\mathbf{v}_{1}\left(\bar{\theta}_{1}, \varphi, \bar{f}_{d, 1}^{\prime}\right)=\mathbf{a}_{t}$ $\left(\bar{f}_{d, 1}^{\prime}\right) \otimes \mathbf{a}_{s}\left(\bar{\theta}_{1}, \varphi\right)=\operatorname{argmax}_{i, j}\left\|\mathbf{v}_{i}^{H}\left(\theta_{i}, \varphi, f_{d, j}^{\prime}\right) \mathbf{e}_{0}\right\|_{2}$. Then, the corresponding eigenvalue and normalized eigenvector can be expressed as

$$
\begin{aligned}
& \mathbf{u}_{1}=\frac{\mathbf{v}_{1}\left(\bar{\theta}_{1}, \varphi, \bar{f}_{d, 1}^{\prime}\right)}{\left\|\mathbf{v}_{1}\left(\bar{\theta}_{1}, \varphi, \bar{f}_{d, 1}^{\prime}\right)\right\|_{2}}, \\
& \lambda_{1}=\mathbf{u}_{1}^{H} \mathbf{e}_{0} .
\end{aligned}
$$

Then, the residual vector between $\mathbf{x}$ and the first normalized eigenvector is $\mathbf{e}_{1}=\mathbf{x}-\lambda_{1} \mathbf{u}_{1}$. For consistently improving the CPNCM estimation accuracy, the iterative procedure will not be stopped until the termination condition $\left\|\mathbf{B}^{H} \mathbf{e}_{q-1}\right\|_{\infty}<\varepsilon$ or the maximum number of iterations is satisfied at the $q_{\max }$-th iteration, where $\varepsilon$ is a constant that represents the threshold and the symbol \|\|$_{\infty}$ represents the infinity norm. At the $q$-th 


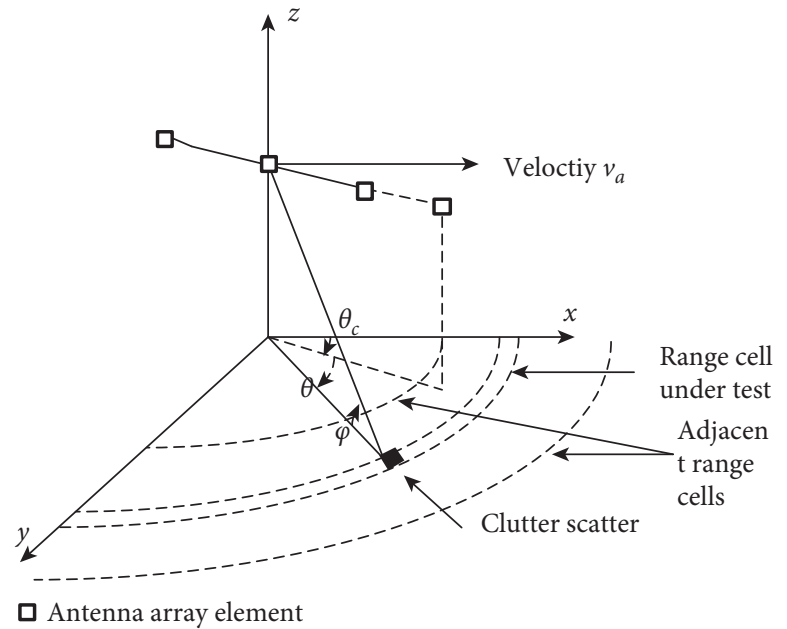

Figure 1: The airborne radar system.
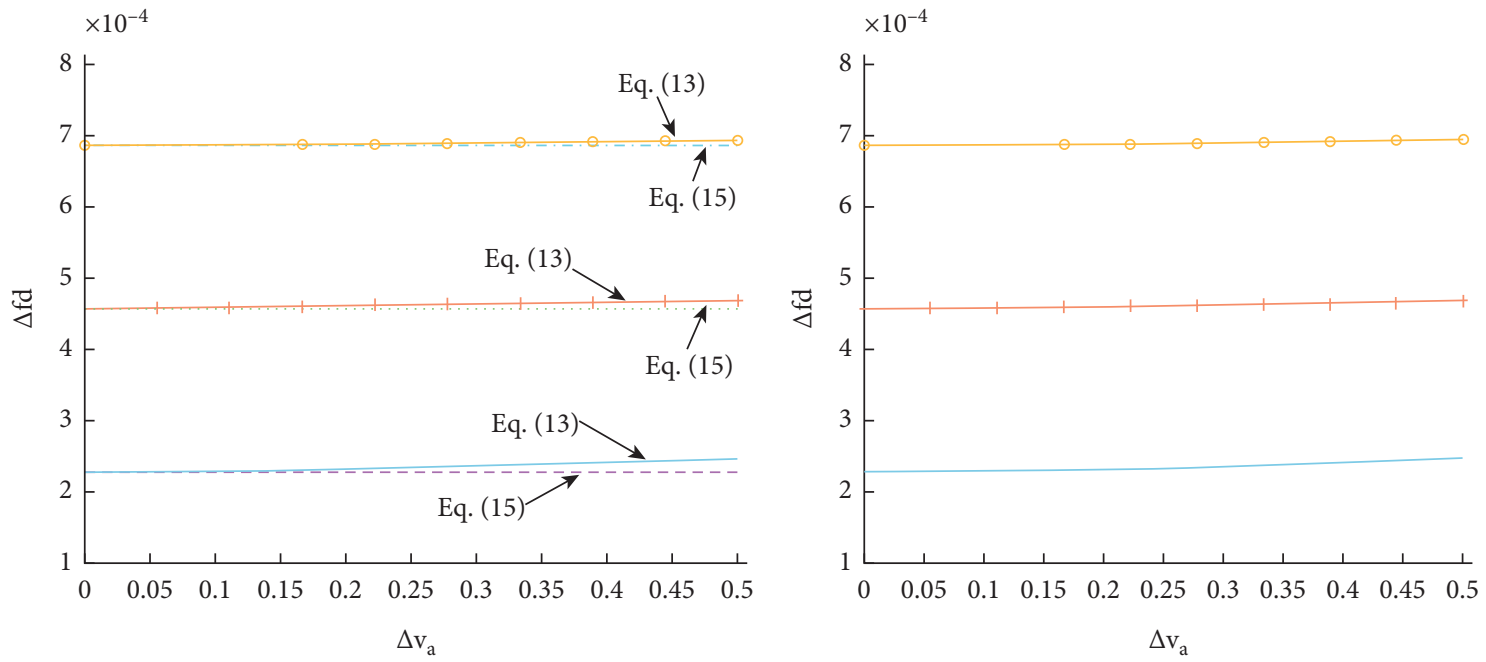

$\begin{array}{lc}-\Delta \theta_{c}=0.5 & \ldots \Delta \theta_{c}=0.5 \\ -\Delta \theta_{c}=1 & \ldots . \Delta \theta_{c}=1 \\ -\Delta \theta_{c}=1.5 & \ldots \Delta \theta_{c}=1.5\end{array}$

(a)

$$
\begin{aligned}
& \text { — } \Delta \theta_{\mathrm{c}}=0.5 \\
& \text { — } \Delta \theta_{\mathrm{c}}=1 \\
& \because \Delta \theta_{\mathrm{c}}=1.5
\end{aligned}
$$

Figure 2: Continued.

(b) 


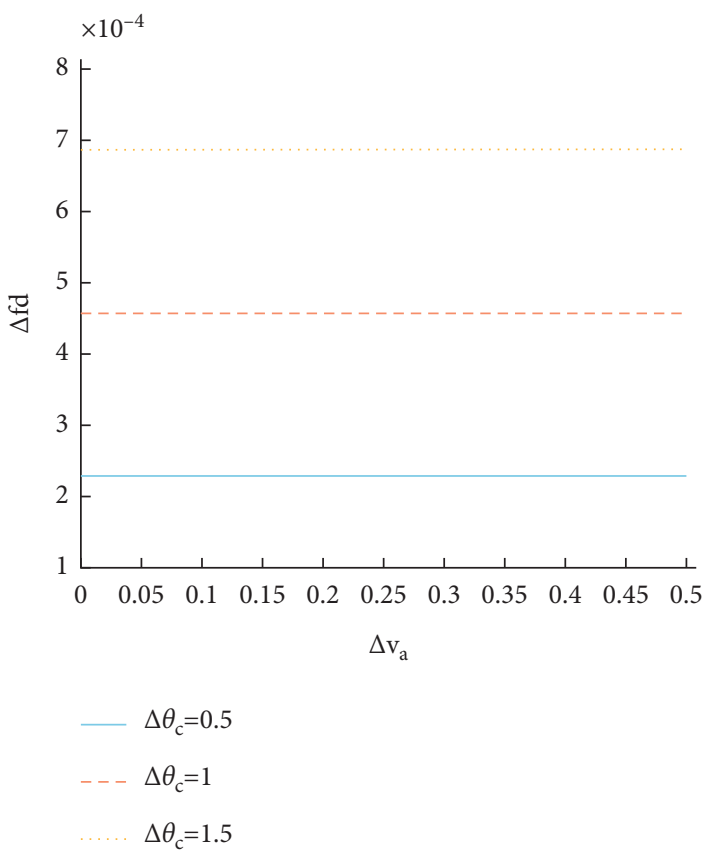

(c)

Figure 2: The Doppler error curve comparison. (a) The Doppler error curves by equations (13) and (15). (b) The Doppler error curve by equation (13). (c) The Doppler error curve by equation (15).

iteration, the most relevant steering-vector is selected as $\mathbf{v}_{q}\left(\bar{\theta}_{q}, \varphi, \bar{f}_{d, q}^{\prime}\right)=\mathbf{a}_{t}\left(\bar{f}_{d, q}^{\prime}\right) \otimes \mathbf{a}_{s}\left(\bar{\theta}_{q}, \varphi\right)=\operatorname{argmax}_{i, j} \| \mathbf{v}_{i}^{H}\left(\theta_{i}, \varphi\right.$, $\left.f_{d, j}^{\prime}\right) \mathbf{e}_{q-1} \|_{2}$. As a consequence, the eigenvalue and the normalized eigenvector at the $q$-th iteration are expressed as

$$
\begin{aligned}
& \mathbf{u}_{q}=\frac{\mathbf{v}_{q}\left(\bar{\theta}_{q}, \varphi, \bar{f}_{d, q}^{\prime}\right)-\sum_{p=1}^{q-1}\left[\mathbf{u}_{p}^{H} \mathbf{v}_{q}\left(\bar{\theta}_{q}, \varphi, \bar{f}_{d, q}^{\prime}\right)\right] \mathbf{u}_{p}}{\left\|\mathbf{v}_{q}\left(\bar{\theta}_{q}, \varphi, \bar{f}_{d, q}^{\prime}\right)-\sum_{p=1}^{q-1}\left[\mathbf{u}_{p}^{H} \mathbf{v}_{q}\left(\bar{\theta}_{q}, \varphi, \bar{f}_{d, q}^{\prime}\right)\right] \mathbf{u}_{p}\right\|_{2}}, \\
& \lambda_{q}=\mathbf{u}_{q}^{H} \mathbf{e}_{q-1} .
\end{aligned}
$$

It is worth noting that $\mathbf{u}_{i}$ and $\mathbf{u}_{j}(i \neq j)$ are mutually orthogonal due to the Schmidt orthogonalization processing in the iteration. For better understanding, the aforementioned algorithm is summarized in Algorithm 1. There exists the contradiction between the performance and computational cost. Intuitively, $N_{c}^{\prime}$ should be large for maintaining the satisfied performance. However, large $N_{c}^{\prime}$ will lead to large computational cost. Fortunately, the rule for choosing $N_{c}^{\prime}$ has been discussed in the previous literatures $[4,27]$, and $N_{c}^{\prime} \geq 4 N$ will assure the performance.

After obtaining the selected steering-vector from $\mathbf{B}$, the robust TDPC can be designed in the similar way as the original TDPC, namely,

$$
\mathbf{D}_{\mathrm{R}}=\left[\mathbf{a}_{s}\left(\bar{\theta}_{1}, \varphi\right), \mathbf{a}_{s}\left(\bar{\theta}_{2}, \varphi\right), \ldots, \mathbf{a}_{s}\left(\bar{\theta}_{q_{\max }}, \varphi\right)\right], \bar{F}_{\mathrm{R}}=\operatorname{diag}\left[\exp \left(\mathrm{j} 2 \pi \bar{f}_{d, 1}^{\prime}\right), \ldots, \exp \left(\mathrm{j} 2 \pi \bar{f}_{d, q_{\max }}^{\prime}\right)\right]
$$

Accordingly, in RTDPC, $\mathbf{A}_{\mathrm{RTDPC}}=\mathbf{D}_{\mathrm{R}} \overline{\mathrm{F}}_{\mathrm{R}} \mathbf{D}_{\mathrm{R}}\left(\mathbf{D}_{\mathrm{R}} \mathbf{D}_{\mathrm{R}}^{H}\right)^{-1}$ and

$$
\mathbf{H}_{\mathrm{RTDPC}}=\left[\begin{array}{cccccc}
\mathbf{A}_{\mathrm{RTDPC}} & -\mathbf{I}_{N} & & & \\
& \mathbf{A}_{\mathrm{RTDPC}} & -\mathbf{I}_{N} & & \mathbf{0} & \\
& & \ddots & \ddots & & \\
& \mathbf{0} & & \mathbf{A}_{\mathrm{RTDPC}} & -\mathbf{I}_{N} & \\
& & & & \mathbf{A}_{\mathrm{RTDPC}} & -\mathbf{I}_{N}
\end{array}\right] \in \mathbf{C}^{(K-1) N \times K N}
$$


Input: $\mathbf{x}, \mathbf{B}$, threshold $\varepsilon$, and maximum iteration number $q_{\max }$

For $q=1,2,3, \ldots, q_{\max }$ do

Update $\mathbf{u}$ and $\lambda$

$\mathbf{v}_{q}\left(\bar{\theta}_{q}, \varphi, \bar{f}_{d, q}^{\prime}\right)=\operatorname{argmax}_{i, j}\left\|\mathbf{v}_{i}^{H}\left(\theta_{i}, \varphi, f_{d, j}^{\prime}\right) \mathbf{e}_{q-1}\right\|_{2}$,

$\mathbf{u}_{q}=\left(\mathbf{v}_{q}\left(\bar{\theta}_{q}, \varphi, \bar{f}_{d, q}^{\prime}\right)-\sum_{p=1}^{q-1}\left[\mathbf{u}_{p}^{H} \mathbf{v}_{q}\left(\bar{\theta}_{q}, \varphi, \bar{f}_{d, q}^{\prime}\right)\right] \mathbf{u}_{p}\right) /\left(\left\|\mathbf{v}_{q}\left(\bar{\theta}_{q}, \varphi, \bar{f}_{d, q}^{\prime}\right)-\sum_{p=1}^{q-1}\left[\mathbf{u}_{p}^{H} \mathbf{v}_{q}\left(\bar{\theta}_{q}, \varphi, \bar{f}_{d, q}^{\prime}\right)\right] \mathbf{u}_{p}\right\|_{2}\right)$ and $\lambda_{q}=\mathbf{u}_{q}^{H} \mathbf{e}_{q-1}$.

Stop if $\left\|\mathbf{B}^{H} \mathbf{e}_{q-1}\right\|_{\infty}<\varepsilon$ or $q>q_{\max }$ is satisfied

Output: $\mathbf{u}_{1}, \mathbf{u}_{2}, \ldots, \mathbf{u}_{q}$ and $\lambda_{1}, \lambda_{t}, \ldots, \lambda_{q} 90$

Algorithm 1: Steering-vector selection algorithm.

\section{Cascaded Structure}

The proposed RTDPC can be exploited as a prefilter before the STM or the classical STAP method to form the two-stage prefilter and detector. In the first stage, RTDPC as the prefilter can effectively filter out the clutter. The second stage, which is STM or STAP, can further suppress the residual clutter and detect the moving target. Here, we will briefly illustrate the two-stage prefilter and detector.

Assume that the spatial-temporal steering-vector of the target is expressed as $\mathbf{s}_{t}=\mathbf{a}_{t} \otimes \mathbf{a}_{s}$, where $\mathbf{a}_{t} \in \mathbf{C}^{K \times 1}$ and $\mathbf{a}_{s} \in \mathbf{C}^{N \times 1}$, respectively, are the temporal and spatial target steering-vectors. After the clutter prefilter by $\tilde{x}=\mathbf{H}_{\mathrm{RTDPC}} \mathbf{x}$, the following moving target detection methods as the second stage are illustrated separately:

(1) STM. The RTDPC prefiltering operation on the target vector $\mathbf{s}$ is

$$
\widetilde{s}_{t}=\mathbf{H}_{\mathrm{RTDPC}} \mathbf{s}_{t} .
$$

Correspondingly, the weight vector of STM is

$$
\mathbf{w}_{s t}=\mathbf{H}_{\mathrm{RTDPC}}\left(\left(\mathbf{a}_{t} \cdot \mathbf{b}_{t}\right) \otimes\left(\mathbf{a}_{s} \cdot \mathbf{b}_{s}\right)\right),
$$

where is the Hadamard product implying the element-by-element operation of two vectors, and $\mathbf{b}_{t} \in \mathbf{C}^{K \times 1}$ and $\mathbf{b}_{s} \in \mathbf{C}^{N \times 1}$ are the temporal and spatial static window vectors adopted for attenuating the sidelobes, respectively.

(2) Dimension-Reduced STAP. Suppose that the corresponding dimension-reduced matrix for STAP is $\mathbf{T} \in \mathbf{C}^{r \times(K-1) N}$, then a dimension-reduced operation is made on the prefiltered data and target steering-vector as follows:

$$
\begin{aligned}
\widehat{x} & =\mathbf{T} \tilde{x}, \\
\widehat{s}_{t} & =\mathbf{T} \widetilde{s}_{t} .
\end{aligned}
$$

As a result, the dimension-reduced space-time filter is as follows:

$$
\widehat{w}=\mu \mathbf{R}_{\bar{x}}^{-1} \widehat{s}_{t}
$$

where $\mu$ is a constant and $\mathbf{R}_{\widehat{x}}=E\left[\widehat{x} \widehat{x}^{H}\right]$ is the correlation matrix of the dimension-reduced data $\hat{x}$. It is often taken place by the maximum likelihood estimation of itself.

\section{Experimental Results}

To start with, the simulation experiments are conducted to verify the performance of RTDPC.

In the simulations, the uniform linear array is $N=8$ antennas with interelement $d=0.1 \mathrm{~m}$. In a CPI, $K=16$ pulses are transmitting at a pulse repetition frequency $f_{r}=2800 \mathrm{~Hz}$. The airborne platform is $h_{a}=9 \mathrm{Km}$ high at a speed of $v_{a}=140 \mathrm{~m} / \mathrm{s}$. The CNR (clutter to noise ratio) is $60 \mathrm{~dB}$. An $\mathrm{SNR}=0 \mathrm{~dB}$ (signal to noise ratio) moving target is injected into the underdetection range cell with Doppler frequency $f_{\mathrm{dr}}=0.2 f_{r}$. The errors for the estimated velocity and crab angle are set to be $\Delta v_{a}=0.5 \mathrm{~m} / \mathrm{s}$ and $\Delta \theta_{c}=1.5^{\circ}$, respectively. The MVDR (minimum variance distortionless response) spectral is exploited to testify the ability of RTDPC. It is defined as $P=$ $\left(1 /\left(\mathbf{s}^{H} \mathbf{R}^{-1} \mathbf{s}\right)\right)[3]$, where [.] ${ }^{-1}$ means the inverse of a matrix, $\mathbf{s}$ is the searching steering-vector, and $\mathbf{R}=\mathbf{R}_{c+n}+\mathbf{R}_{s}$ is the covariance of clutter plus noise and target.

In Figure 3, a target with normalized Doppler frequency $\left(f_{d} / f_{r}\right)=0.2$ is injected near the main clutter region. Figure 3(a) shows the original clutter spectral. The clutter ridge is distributed along the diagonal in the angle-Doppler plane. Compared with the strong clutter, the target which is located on the right side of the clutter ridge is much weaker. Figures 3(b) and 3(c) show, with accurate prior knowledge, the clutter spectral after TDPC and RTDPC as clutter prefilters. Obviously, the energy of the moving target is considerably stronger than the main clutter. This result indicates that both TDPC and RTDPC can effectively suppress the clutter by forming the nulling along the clutter ridge and maintain the target constant when the prior knowledge is accurate.

Figures 4-6 show the clutter filtering performance of TDPC and RTDPC when the prior knowledge is not accurate. It is clearly demonstrated that when crab angle is inaccurate, the SNR will be considerably degraded by TDPC. However, by RTDPC, the SNR almost remains the same as in Figure 3(c) and the clutter is also effectively filtered out. Moreover, as the analysis in Section 3, inaccurate crab angle influences the performances of TDPC more than inaccurate velocity.

In Figures 7 and 8, the robust performance of RTDPC is verified under nonsidelooking scenario. For the variation of crab angle, the clutter ridge is distributed as a half-ellipse in the angle-Doppler plane, which implies the nonlinear relationship between the Doppler frequency and the spatial frequency. Under this condition, RTDPC can still effectively filter the clutter and maintain the SNR constant. However, as in the sidelooking scenario, TDPC considerably degrades the SNR of 


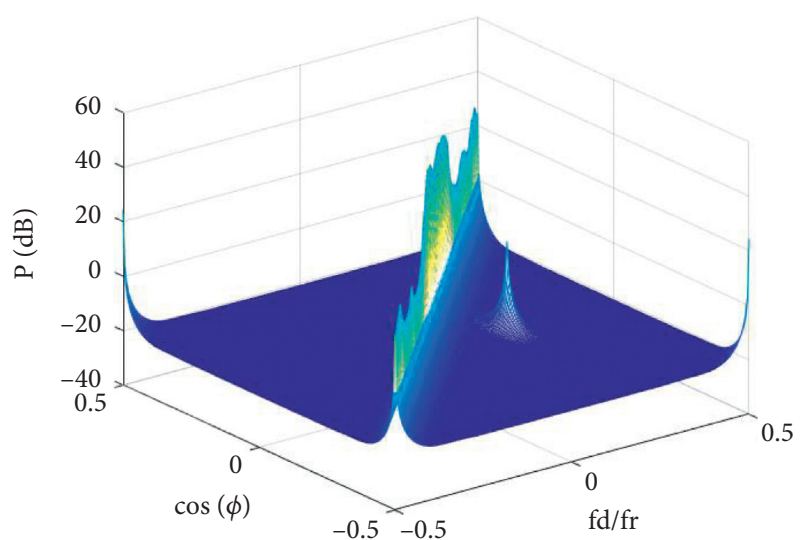

(a)

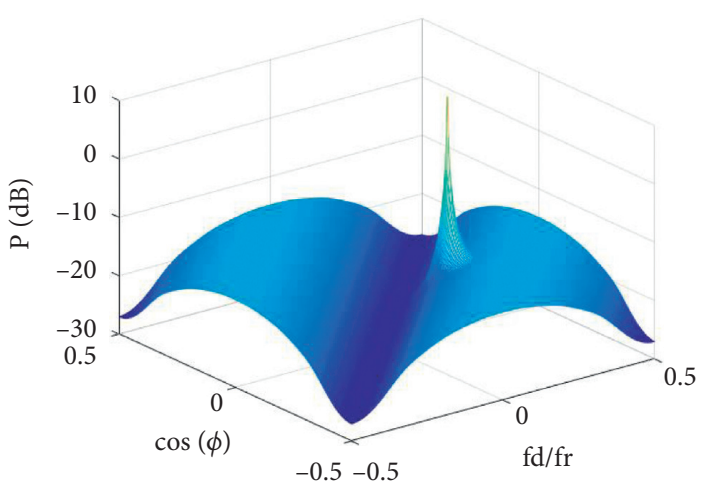

(b)

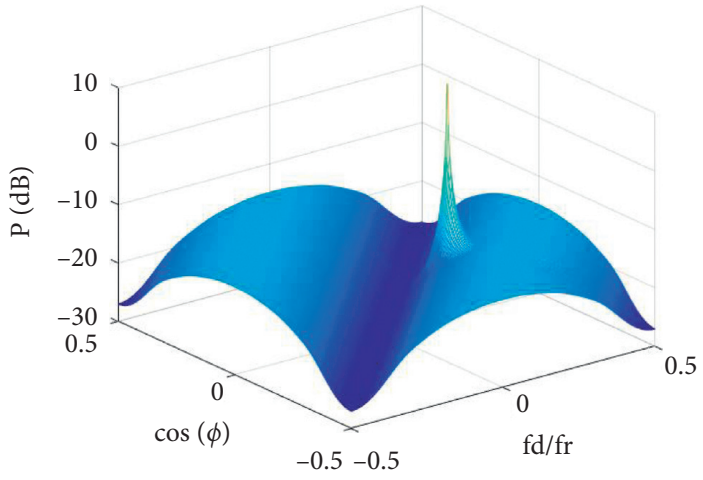

(c)

FIgURE 3: The clutter spectral before and after the prefiltering $\left(\theta_{c}=0^{\circ}\right)$ : (a) the original clutter spectral; (b) the clutter spectral after TDPC filtering; (c) the clutter spectral after RTDPC filtering.

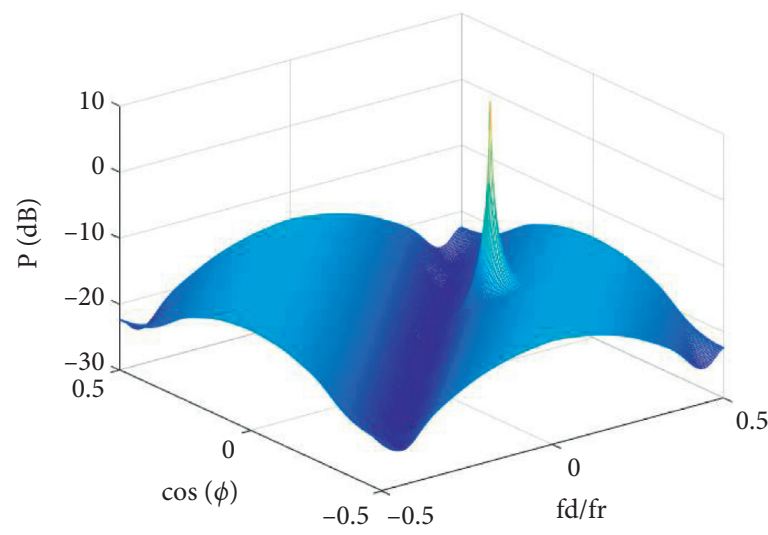

(a)

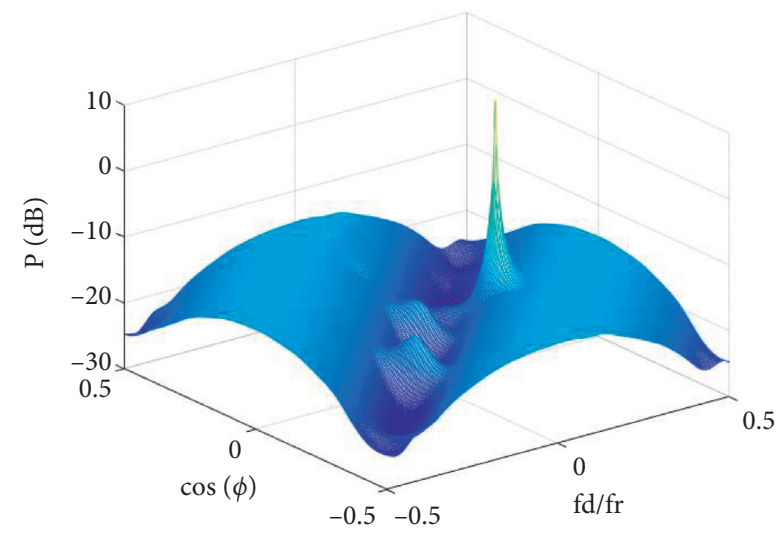

(b)

Figure 4: The clutter spectral after the prefiltering with $\Delta v_{a}=0.5 \mathrm{~m} / \mathrm{s}\left(\theta_{c}=0^{\circ}\right)$ : (a) the clutter spectral after TDPC filtering; (b) the clutter spectral after RTDPC filtering.

target when the prior knowledge is inaccurate. From the simulation results, we can conclude that although TDPC can effectively suppress the clutter when the prior knowledge is not accurate, the target is also impaired, which is detrimental to the following target detection.

Real Data. In order to verify a good many achievements in the theory of STAP, MCARM program was established by Rome Laboratory and Northrop Grumman Corporation [37]. The MCARM data used in the experiment come from acquisition 575 on flight 5 (rl050575). The operating parameters used to obtain the data are as follows: the platform velocity $v_{a}=100.2 \mathrm{~m} / \mathrm{s}$, the crab angle $\theta_{c}=7.28^{\circ}$, the array element spacing $d=0.1092 \mathrm{~m}$, the radar operating wavelength $\lambda=0.2419 \mathrm{~m}$, and the 


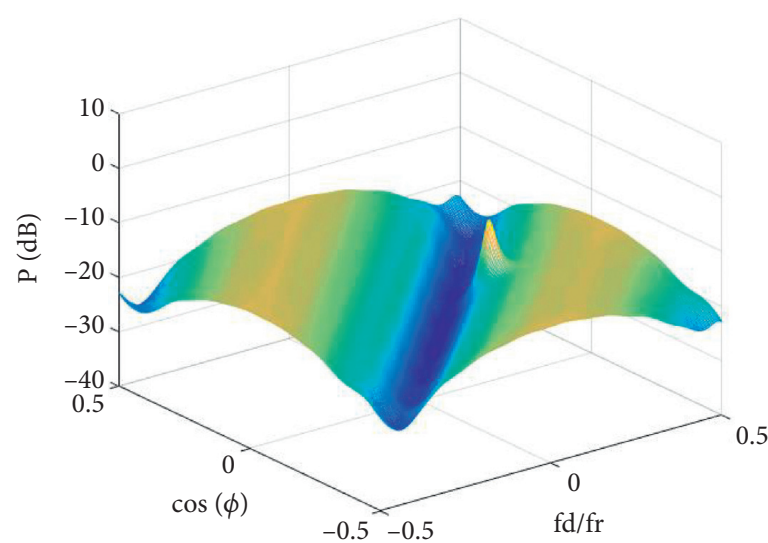

(a)

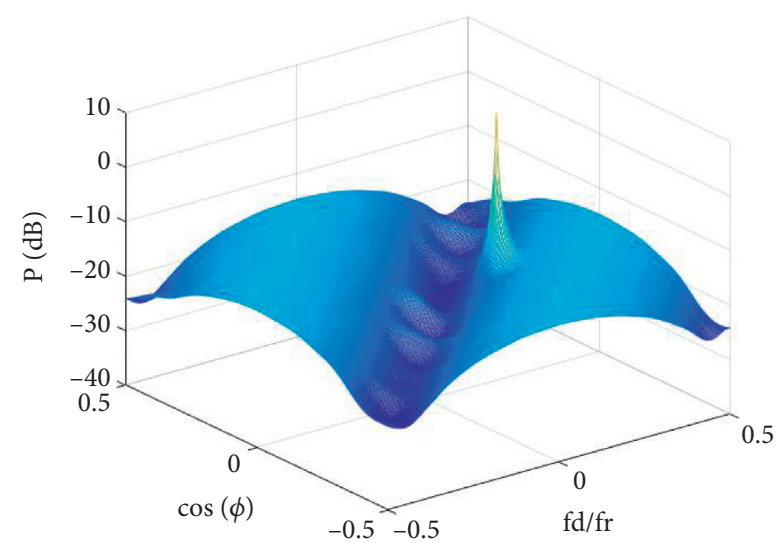

(b)

Figure 5: The clutter spectral after the prefiltering with $\Delta \theta_{c}=1.5^{\circ}\left(\theta_{c}=0^{\circ}\right)$ : (a) the clutter spectral after TDPC filtering; (b) the clutter spectral after RTDPC filtering.

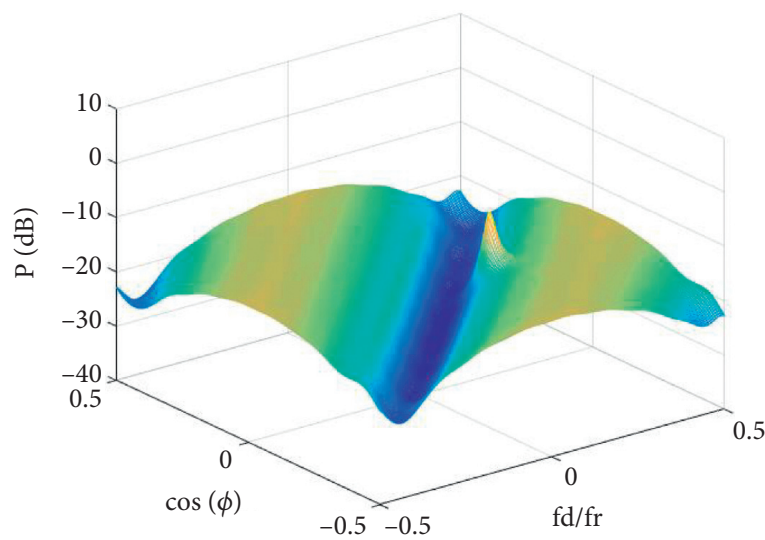

(a)

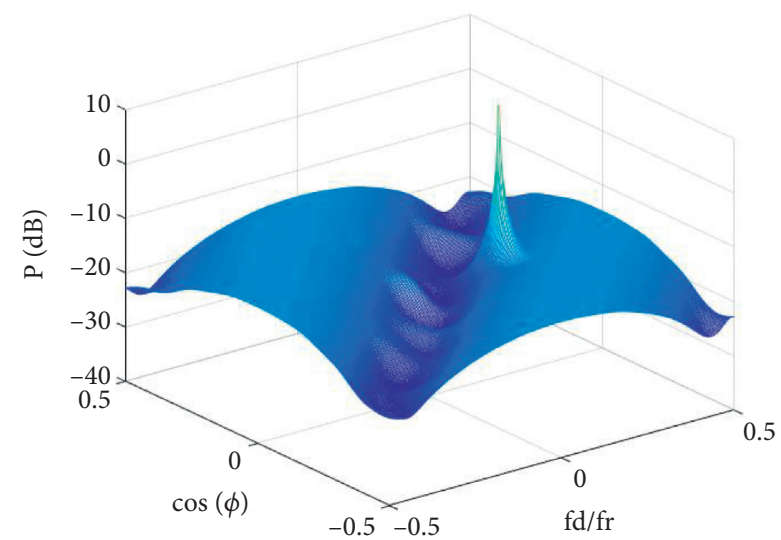

(b)

FIGURE 6: The clutter spectral after the prefiltering with $\Delta v_{a}=0.5 \mathrm{~m} / \mathrm{s}$ and $\Delta \theta_{c}=1.5^{\circ}\left(\theta_{c}=0^{\circ}\right)$ : (a) the clutter spectral after TDPC filtering; (b) the clutter spectral after RTDPC filtering.

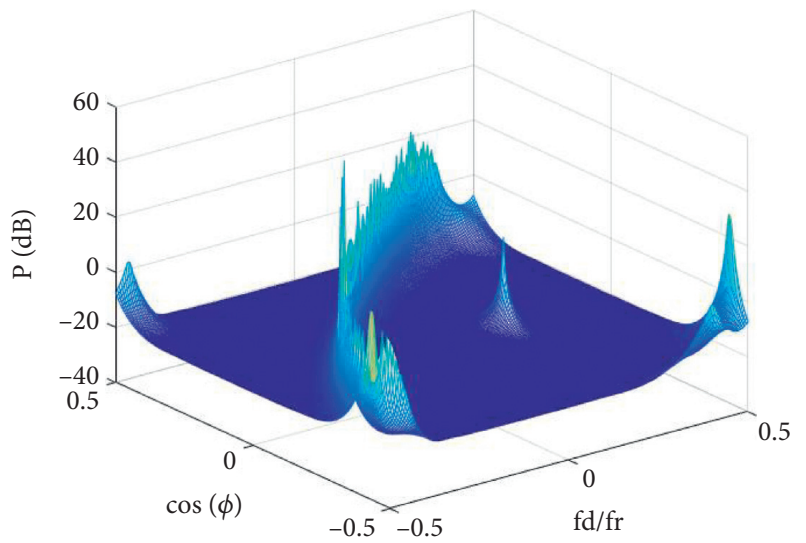

(a)

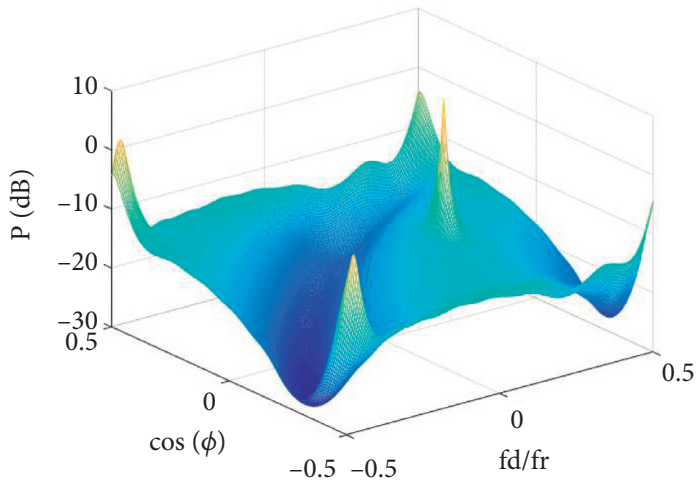

(b)

Figure 7: Continued. 


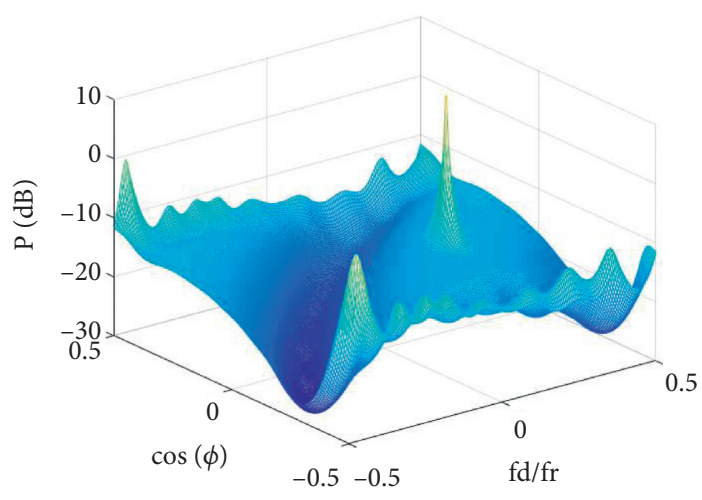

(c)

Figure 7: The clutter spectral before and after the prefiltering $\left(\theta_{c}=30^{\circ}\right)$ : (a) the original clutter spectral; (b) the clutter spectral after TDPC filtering; (c) the clutter spectral after RTDPC filtering.

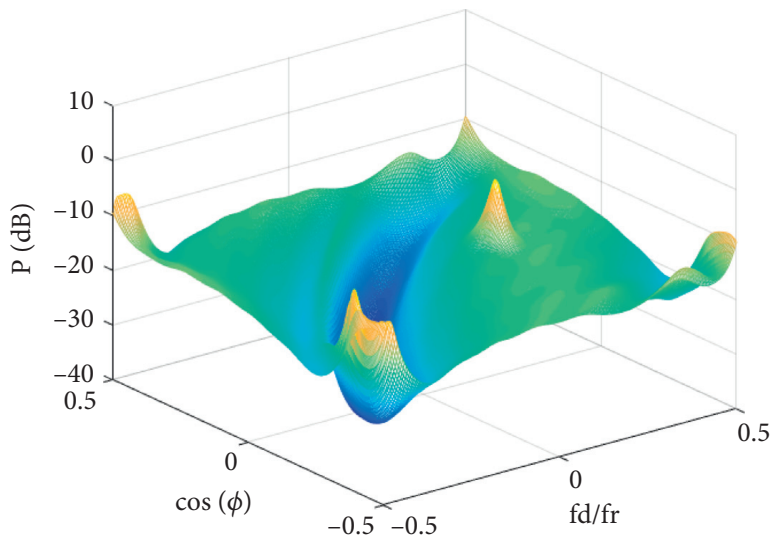

(a)

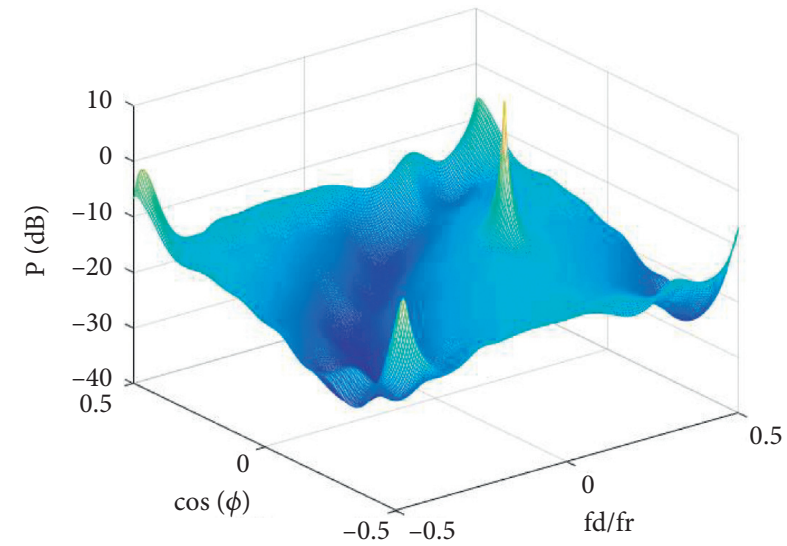

(b)

FIGURE 8: The clutter spectral after the prefiltering with $\Delta v_{a}=0.5 \mathrm{~m} / \mathrm{s}$ and $\Delta \theta_{c}=1.5^{\circ}\left(\theta_{c}=30^{\circ}\right)$ : (a) the clutter spectral after TDPC filtering; (b) the clutter spectral after RTDPC filtering.

pulse repetition frequency $f_{r}=1984 \mathrm{~Hz}$. As in the simulated experiments, the estimated errors in TDPC and RTDPC for velocity and crab angle are also set to be $\Delta v_{a}=$ $0.5 \mathrm{~m} / \mathrm{s}$ and $\Delta \theta_{c}=1.5^{\circ} .201-500$ range bins are selected to verify the proposed algorithm. The first 11 azimuth channels and the first 32 pulses are exploited here. Two $\mathrm{SCR}=-20 \mathrm{~dB}$ (signal to clutter ratio) moving targets with azimuth angles $90^{\circ}$ and Doppler frequency $f_{d}=-0.157 f_{r}$ are separately injected near the clutter main beam at the range bins 350 and 400 .

Figure 9 shows that the normalized output power (NOP) of each range bin by making use of TDPC + STM and RTDPC + STM. The average NOPs below the target of them are about $19.29 \mathrm{~dB}, 25.87 \mathrm{~dB}$, and $34.93 \mathrm{~dB}$, respectively. It is clearly demonstrated that after RTDPC filtering, STM can identify the two weak targets from the residual clutter. However, due to the inaccurate prior knowledge, after TDPC filtering, the clutter energy is still strong. Similarly, as shown in Figures 10 and 11, the average NOPs below the target for FA, TDPC + FA, and RTDPC + FA are about $33.48 \mathrm{~dB}, 41.43 \mathrm{~dB}$, and $43.38 \mathrm{~dB}$ and those for EFA, TDPC + EFA, and RTDPC + EFA are about $45.33 \mathrm{~dB}, 45.86 \mathrm{~dB}$, and $46.71 \mathrm{~dB}$. The results infer that the STAP method followed RTDPC can achieve relatively good performance at the improvement of output SCNR and the corresponding detection performance. 


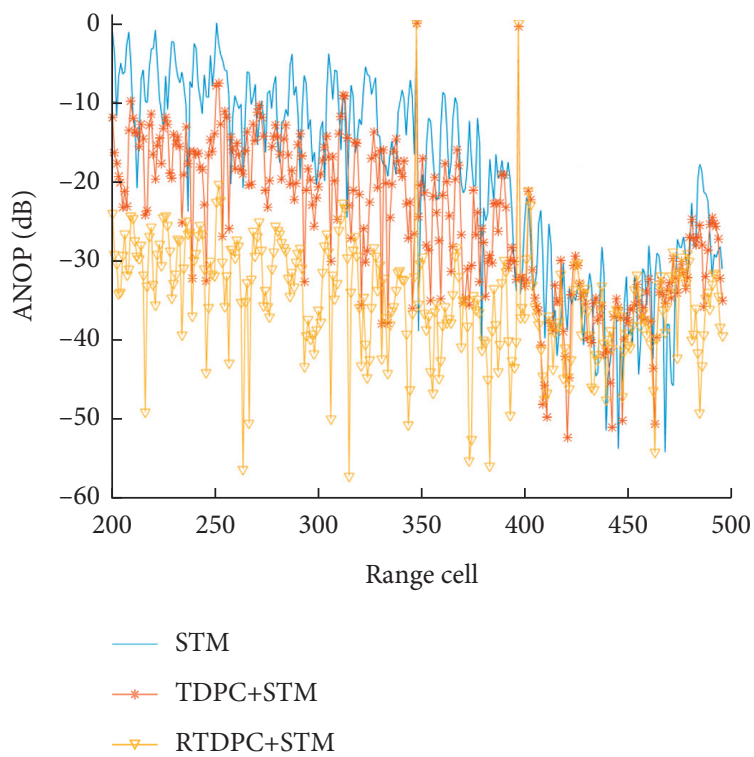

Figure 9: Curves of NOP against range bins for STM, TDPC + STM, and RTDPC + STM.

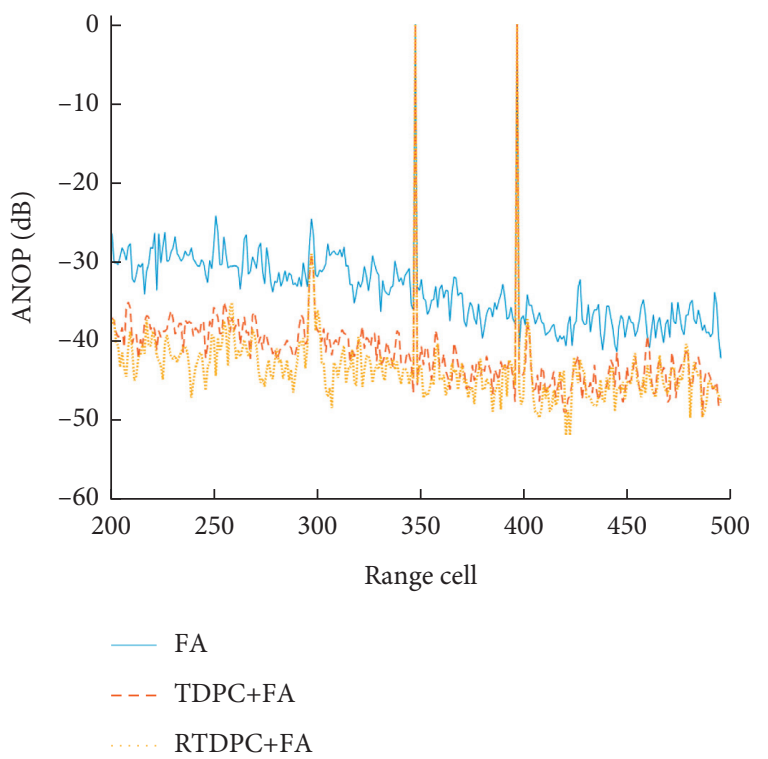

FIgURE 10: Curves of NOP against range bins for FA, TDPC + FA, and RTDPC + FA. 


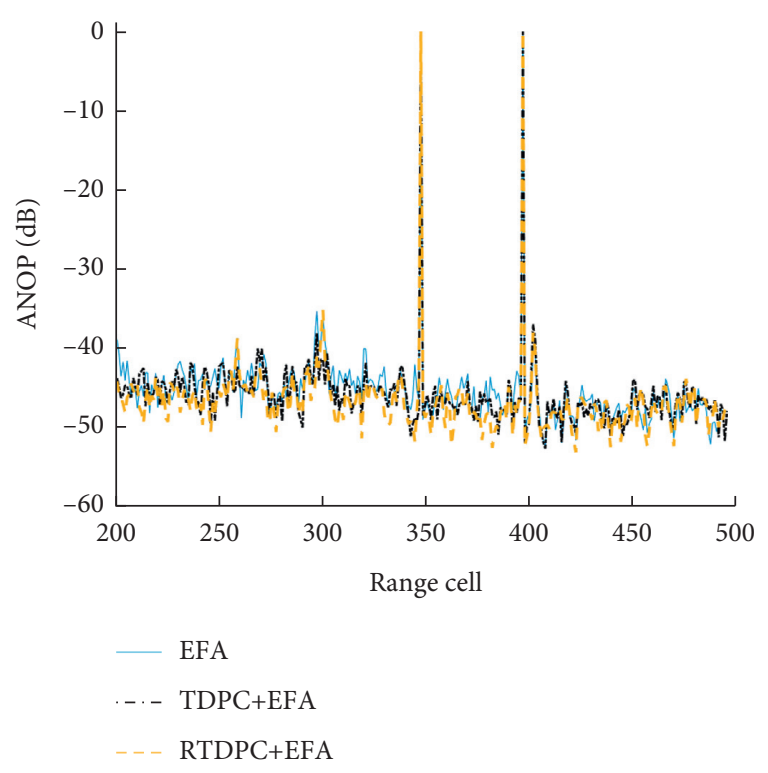

FIgURE 11: Curves of NOP against range bins for EFA, $\mathrm{TDPC}+\mathrm{EFA}$, and RTDPC + EFA.

\section{Conclusions}

The effectiveness of TDPC depends largely on the accuracy of estimated radar parameters. The estimation errors of platform velocity and crab angle in practice caused by the complicated airborne flight environment influence the clutter suppression ability of TDPC. RTDPC that adds the errors of platform velocity and crab angle into the design of filtering coefficient matrix for increasing the TDPC"s applicability is proposed in this paper. Both the simulated and the MCARM real data are utilized to compare the clutter suppression performance of RTDPC with that of TDPC under the condition of inaccurate platform velocity and crab angle. The moving target detectability of following STAP algorithms is also enhanced after RTDPC as the filter.

\section{Data Availability}

All the data used in this work are based on simulations in MATLAB and are available for any research work.

\section{Conflicts of Interest}

The authors declare that there are no conflicts of interest.

\section{Acknowledgments}

This work was sponsored by National Natural Science Foundation of China under Grants 61901371, 61901372, and 51974250 , in part by the Natural Science Basic Research Program of Shaanxi under Grants 2020JQ-600 and 2020JQ599 , and in part by the Youth Science and Technology Nova Project in Shaanxi Province under Grant 2020KJXX-018.

\section{References}

[1] L. E. Brennan and L. S. Reed, "Theory of adaptive radar," IEEE Transactions on Aerospace and Electronic Systems, vol. 9, no. 2, pp. 237-252, 1973.

[2] W. L. Melvin, "A STAP overview," IEEE Aerospace and Electronic Systems Magazine, vol. 19, no. 1, pp. 19-35, 2004.

[3] J. R. Guerci, Space-Time Adaptive Processing for Radar, Artech House, Norwood, MA, USA, 2003.

[4] R. Klemm, Principles of Space-Time Adaptive Processing, Institute of Electrical Engineering, London, UK, 2006.

[5] R. Klemm, "Adaptive airborne MTI: an auxiliary channel approach," IEEE Proceedings F Communications, Radar and Signal Processing, vol. 134, no. 3, pp. 269-276, 1987.

[6] H. Hong Wang and L. Lujing Cai, "On adaptive spatialtemporal processing for airborne surveillance radar systems," IEEE Transactions on Aerospace and Electronic Systems, vol. 30, no. 3, pp. 660-670, 1994.

[7] R. C. Dippetro, "Extended factored space-time processing for airborne radar system," in Proceedings of the 1992 Conference Record of the Twenty-Sixth Asilomar Conference on Signals, Systems \& Computers, pp. 425-430, Pacific Grove, CA, USA, October 1992.

[8] Z. Bao, G. Liao, R. Wu, Y. Zhang, and Y. Wang, "Adaptive spatial-temporal processing for airborne radars," Chinese Journal of Electronics, vol. 2, no. 1, pp. 2-7, 1993.

[9] Y. Zhou, D.-Z. Feng, G.-H. Zhu, and W.-K. Nie, "The postDoppler adaptive processing method based on the spatial domain reconstruction," Signal Processing, vol. 111, pp. 89-93, 2015.

[10] Y. Zhou, L. Wang, X. Chen, C. Wen, B. Jiang, and D. Fang, "An improving EFA for clutter suppression by using the persymmetric covariance matrix estimation," Circuits, Systems, and Signal Processing, vol. 37, no. 2, pp. 1-14, 2018.

[11] Y. Zhou, L. Wang, Y. Li, C. Wen, and B. Jiang, "A fast iterative three-dimensional joint domain localized method in airborne MIMO radar," Circuits, Systems, and Signal Processing, vol. 38, no. 7, pp. 3370-3383, 2019.

[12] K. Duan, H. Xu, H. Yuan, H. Xie, and Y. Wang, "ReducedDOFs three-dimension STAP via subarray synthesis for nonsidelooking planar array airborne radar," IEEE Transactions on Aerospace and Electronic Systems, vol. 56, no. 4, pp. 3311-3325, 2020.

[13] A. M. Haimovich and Y. Bar-Ness, "An eigenanalysis interference canceler," IEEE Transactions on Signal Processing, vol. 39, no. 1, pp. 76-84, 1991.

[14] A. M. Haimovich, "Asymptotic distribution of the conditional signal-to-noise ratio in an eigenanalysis-based adaptive array," IEEE Transactions on Aerospace and Electronic Systems, vol. 33, no. 3, pp. 988-997, 1997.

[15] C. D. Peckham, A. M. Haimovich, T. F. Ayoub, J. S. Goldstein, and I. S. Reid, "Reduced-rank STAP performance analysis," IEEE Transactions on Aerospace and Electronic Systems, vol. 36, no. 2, pp. 664-676, 2000.

[16] J. S. Goldstein and I. S. Reed, "Subspace selection for partially adaptive sensor array processing," IEEE Transactions on Aerospace and Electronic Systems, vol. 33, no. 2, pp. 539-544, 2002.

[17] R. Fa, R. C. de Lamare, and L. Wang, "Reduced-rank stap schemes for airborne radar based on switched joint interpolation, decimation and filtering algorithm," IEEE Transactions on Signal Processing, vol. 58, no. 8, pp. 4182-4194, 2010. 
[18] S. Zhu, G. Liao, J. Xu, and Y. Gao, "Robust space-time adaptive processing with colored loading using iterative optimization," Digital Signal Processing, vol. 35, no. C, pp. 14-20, 2014.

[19] W. Feng, Y. Zhang, and X. He, "Complexity reduction and clutter rank estimation for MIMO-phased STAP radar with subarrays at transmission," Digital Signal Processing, vol. 60, pp. 296-306, 2017.

[20] X. Wang, Z. Yang, J. Huang, and R. C. de Lamare, "Robust Two-stage reduced-dimension sparsity-aware STAP for airborne radar with coprime arrays," IEEE Transactions on Signal Processing, vol. 68, pp. 81-96, 2019.

[21] Y. Wang, W. Liu, W. Xie, and Y. Zhao, "Reduced-rank spacetime adaptive detection for airborne radar," Science China Information Sciences, vol. 57, no. 8, pp. 1-11, 2014.

[22] W. Liu, J. Liu, C. Han, Y. Gao, and Y. L. Wang, "Multichannel adaptive signal detection: basic theory and literature review," Science China-Information Sciences, 2021.

[23] S. Haykin, "Cognitive radar: a way of the future," IEEE Signal Processing Magazine, vol. 23, no. 1, pp. 30-40, 2006.

[24] J. R. Guerci and E. J. Baranoski, "Knowledge-aided adaptive radar at DARPA: an overview," IEEE Signal Processing Magazine, vol. 23, no. 1, pp. 41-50, 2006.

[25] P. Stoica, L. Jian Li, X. Xumin Zhu, and J. R. Guerci, "On using a priori knowledge in space-time adaptive processing," IEEE Transactions on Signal Processing, vol. 56, no. 6, pp. 2598-2602, 2008.

[26] J. Xu, G. Liao, S. Zhu, and L. Huang, "Response vector constrained robust LCMV beamforming based on semidefinite programming," IEEE Transactions on Signal Processing, vol. 63, no. 21, pp. 5720-5732, 2015.

[27] Z. Yang and R. C. de Lamare, "Enhanced knowledge-aided space-time adaptive processing exploiting inaccurate prior knowledge of the array manifold," Digital Signal Processing, vol. 60, pp. 262-276, 2017.

[28] Y. Zhao, S. Wan, S. Lu, J. Sun, and P. Lei, "Exploiting the persymmetric property of covariance matrices for knowledgeaided space-time adaptive processing," IEEE Access, vol. 6, no. 1, pp. 68001-68012, 2018.

[29] Z. Li, Y. Zhang, H. Liu, B. Xue, and Y. Liu, “A robust STAP method for airborne radar based on clutter covariance matrix reconstruction and steering vector estimation," Digital Signal Processing, vol. 78, pp. 82-91, 2018.

[30] M. Riedl and L. C. Potter, "Knowledge-aided Bayesian spacetime adaptive processing," IEEE Transactions on Aerospace and Electronic Systems, vol. 54, no. 4, pp. 1850-1861, 2018.

[31] T. Fu, T. Wang, J. Wu, and Y. Su, "A knowledge aided SPICE space time adaptive processing method for airborne radar with conformal array," Signal Processing, vol. 152, pp. 54-62, 2018.

[32] F. Tao, T. Wang, J. Wu, and X. Lin, "A novel KA-STAP method based on Mahalanobis distance metric learning," Digital Signal Processing, vol. 97, Article ID 102613, 2020.

[33] P. He, S. He, Z. Yang, and P. Huang, "An off-grid STAP algorithm based on local mesh splitting with bistatic radar system," IEEE Signal Processing Letters, vol. 27, pp. 1355-1359, 2020.

[34] X.-M. Li, D.-Z. Feng, H.-W. Liu, M.-D. Xing, and D. Luo, "Two-dimensional pulse-to-pulse canceller of ground clutter in airborne radar," IET Radar, Sonar \& Navigation, vol. 3, no. 2, pp. 133-143, 2009.

[35] Y. Cao, D. Feng, P. Shui, and C. Xiang, "Two-dimensional pulseto-pulse canceller applied in multiple-input-multiple-output radar," IET Radar Sonar Navigation, vol. 6, no. 9, pp. 945-955, 2013.

[36] J. A. Tropp and A. C. Gilbert, "Signal recovery from random measurements via orthogonal matching pursuit," IEEE Transactions on Information Theory, vol. 53, no. 2, pp. 4655-4666, 2007.

[37] D. Sloper, D. Fenner, J. Arntz, and E. Fogle, "Multi-Channel airborne radar measurement (MCARM), MCARM flight test," in Proceedings of the IEEE Radar Conference, Florence, Italy, April 1996. 